\title{
Confinement from constant field condensates
}

\author{
Patricio Gaete* \\ Departamento de Física, Universidad Técnica F. Santa María, Valparaíso, Chile \\ Eduardo Guendelman ${ }^{\dagger}$ \\ Physics Department, Ben Gurion University, Beer Sheva 84105, Israel \\ Euro Spallucci ${ }^{\ddagger}$ \\ Dipartimento di Fisica Teorica, Università di Trieste and INFN, Sezione di Trieste, Italy
}

(Dated: September 14, 2018)

\begin{abstract}
For a $(2+1)$-dimensional reformulated $S U(2)$ Yang-Mills theory, we compute the interaction potential within the framework of the gauge-invariant but path-dependent variables formalism. This reformulation is due to the presence of a constant gauge field condensate. Our results show that the interaction energy contains a linear term leading to the confinement of static probe charges. This result is equivalent to that of the massive Schwinger model.
\end{abstract}

PACS numbers: 11.10.Ef, 11.10.Kk

\section{INTRODUCTION}

One of the most important problems facing $Q C D$ in the infrared region is the confinement of quarks. One expects that the gluon field exchanged between a quark and an antiquark gathers up into a string, thus preventing the quarks from separating to large distances. As is well known, directly observable are only colorless formations of a quark and antiquark (mesons). There is, of course, no known way to analytically derive confinement from first principles up to now. Nevertheless some evidence has been obtained at the level of computer simulations based on lattice gauge theories [1].

In this context, it may be recalled that many authors have linked the confinement phenomena to the instability of the perturbative $Q C D$ vacuum [2,3], which indicates the existence of condensates of gauge fields. Confinement as a consequence of the interaction between a constant chromo-magnetic background and the axion field has been recently discussed in [4].

The need for constant condensates of gauge fields appears already evident in the loop expansion for a $(2+1)$ dimensional gauge theory [5]. This is because in $Q C D_{3}$, being a superrenormalizable theory, the loop expansion probes deeper and deepen into the infrared region at every increasing loop order. In particular, in order to cancel all leading infrared divergences it becomes necessary to introduce constant gauge field potentials (which are not pure gauge in the non-Abelian theory) into the vacuum. An appropriate gaussian averaging over the field strengths and directions is required as well (here the gaussian width in the averaging must be taken to zero to achieve cancellation of infrared divergences) [5].

On the other hand, in Ref. [6] a qualitative analysis of the effect of these constant gauge fields was started and the arguments presented there indicated that the constant gauge fields induce linear potentials between static charges. In fact, it was proposed a reformulation of pure $S U(2)$ Yang-Mills theory in terms of new variables suitable for its low energy content. In terms of these variables the long distance physics is described by an effective action which is $U(1)$ gauge theory. The idea that confinement is basically an abelian effect can be dated back to [7]. More recently it has been related to relativistic membrane dynamics in [8], and implemeted through the abelian projection method in [9]. Some peculiar quantum aspects of the effective long range dynamics of QCD, and certain intriguing analogies with the Schwinger model, has been discussed in [10]. Clearly, in order to put our qualitative analysis into a firmer footing, one needs an explicit calculation for the interaction energy. In this Letter we address this question and show that $S U(2)$ Yang-Mills theory can be further mapped into the massive Schwinger model [11, 12]. Our calculation is based on the gauge-invariant but path-dependent variables formalism [13]. According to this formalism, the interaction potential between two static charges is obtained once a judicious identification of the physical degrees of freedom is made. It

\footnotetext{
*Electronic address: patricio.gaete@usm.cl

${ }^{\dagger}$ Electronic address: guendel@bgumail.bgu.ac.il

$\ddagger$ Electronic address: euro@ts.infn.it
} 
also offers an alternative technique for determining the static potential for a gauge theory. The novel ingredient we introduce in this letter is the computation of the static potential in the presence of one, or more, compact spacelike dimension. In this sort of Kaluza-Klein approach the limit of infinite radius of compactification can be performed provided a self-consistency condition between the ultraviolet cut-off and the amplitude of the constant background field is satisfied.

\section{INTERACTION ENERGY}

As already stated, our principal purpose is to calculate explicitly the interaction energy between static pointlike sources for three-dimensional $S U(2)$ Yang-Mills theory. To this end we will calculate the expectation value of the energy operator $H$ in the physical state $|\Phi\rangle$, which we will denote by $\langle H\rangle_{\Phi}$. However, before going to the derivation of the interaction energy, we will describe very briefly the new set of variables which map $S U(2)$ Yang-Mills theory to the Abelian gauge model. We take as starting point the three-dimensional space-time Lagrangian:

$$
\mathcal{L}=-\frac{1}{4} \operatorname{Tr}\left(G_{\mu \nu} G^{\mu \nu}\right)=-\frac{1}{4} G_{\mu \nu}^{a} G^{a \mu \nu} .
$$

Here $A_{\mu}(x)=A_{\mu}^{a}(x) T^{a}$, where $T^{a}$ is a hermitian representation of the semi-simple and compact gauge group; and $G_{\mu \nu}^{a}=\partial_{\mu} A_{\nu}^{a}-\partial_{\nu} A_{\mu}^{a}+g \epsilon^{a b c} A_{\mu}^{b} A_{\nu}^{c}$, with $\epsilon^{a b c}$ the structure constants of the $S U(2)$ gauge group.

We should now consider the reformulated version of this theory, which has been developed and analyzed in [6], where one can find the motivations to consider it and details of the change of variables are given. Applying the change of variables defined by the fields $[6]: W_{\mu}^{ \pm}=1 / \sqrt{2}\left[A_{\mu}^{(1)} \pm\left(-i A_{\mu}^{(2)}\right)\right]$ and $A_{\mu} \equiv A_{\mu}^{(3)}$, on (1), one obtains

$$
\mathcal{L}=-\frac{1}{4} F_{\mu \nu} F^{\mu \nu}-\frac{1}{2}\left|D_{\mu} W_{\nu}^{+}-D_{\nu} W_{\mu}^{-}\right|^{2}+\frac{g}{2} F_{\mu \nu} W_{\rho}^{-} S^{\mu \nu \rho \sigma} W_{\sigma}^{-}-\frac{g^{2}}{4} W_{\rho}^{-} S^{\mu \nu \rho \sigma} W_{\sigma}^{+} W^{-\alpha} S_{\mu \nu \alpha \beta} W^{+\beta}
$$

where $F_{\mu \nu}=\partial_{\mu} A_{\nu}-\partial_{\nu} A_{\mu}, D_{\mu}=\partial_{\mu}-i g A_{\mu}$ and $S_{\mu \nu \rho \sigma}=i\left(g_{\mu \rho} g_{\nu \sigma}-g_{\mu \sigma} g_{\nu \rho}\right)$. Next, according to Ref. [6], we restrict our attention to the ground state given by:

$$
\left\{\begin{array}{l}
A_{\mu}^{(1)}=C(\text { constant }) \delta_{\mu}^{1} \\
A_{2}^{(1)}=A_{0}^{(1,2)}=0
\end{array}\right\} \Rightarrow\left\langle W_{\mu}^{+}\right\rangle=\left\langle W_{\mu}^{-}\right\rangle=\frac{C}{\sqrt{2}} \delta_{\mu 1}
$$

Hence the Lagrangian (1) reduces to:

$$
\mathcal{L}=-\frac{1}{4} F_{\mu \nu} F^{\mu \nu}+\frac{1}{2} m^{2} A_{\mu} A^{\mu}-A_{0} J^{0}
$$

where $J^{0}$ is the external current, and $g^{2} C^{2} \equiv m^{2}$. In this Lagrangian we have considered only sources and gauge field excitations in the third direction of $S U(2)$ only. This appears to be enough in order to study the basic features of confinement, as we will show now. Thus the Lagrangian (1) becomes a Maxwell theory supplemented by the Proca term. Notwithstanding, in order to put our discussion into context it is useful to summarize the relevant aspects of the canonical quantization of this reformulated theory from the Hamiltonian point of view.

We now proceed to obtain the Hamiltonian. The canonical momenta read $\Pi^{\mu}=-F^{0 \mu}$, which results in the usual primary constraints $\Pi_{0}=0$, and $\Pi^{i}=F^{i 0}$. Standard techniques for constrained systems then lead to the following canonical Hamiltonian

$$
H_{C}=\int d^{2} x\left\{-A_{0}\left(\partial_{i} \Pi^{i}+m^{2}-J^{0}\right)-\frac{1}{2} \Pi_{i} \Pi^{i}+\frac{1}{4} F_{i j} F^{i j}-m^{2} A_{i} A^{i}\right\} .
$$

Requiring the primary constraint to be stationary, we obtain

$$
\Gamma \equiv \partial_{i} \Pi^{i}+m^{2} A^{0}-J^{0}=0
$$

It can be easily checked that both constraints are second class. This result is not surprising, it explicitly reflects the breaking of the gauge invariance of the theory under consideration. Consequently, special care has to be exercised since it is the gauge invariance that generally establish unitarity and renormalizability in most quantum field theoretical models. To convert the second class system into first class we will adopt the procedure described in Refs.[14, 15]. In 
this way the new system still has the basic features of the original one and has reobtained the gauge symmetry. As stated in Refs. [14-16], we enlarge the original phase space by introducing a canonical pair of fields $\theta$ and $\Pi_{\theta}$. It follows, therefore, that a new set of first class constraints can be defined in this extended space:

$$
\Lambda_{1} \equiv \Pi_{0}+m^{2} \theta=0
$$

and

$$
\Lambda_{2} \equiv \Gamma+\Pi_{\theta}=0
$$

It is easy to verify that the new constraints are first class and in this way restore the gauge symmetry of the theory under consideration. It is to be observed that the $\theta$ fields only enlarge the unphysical sector of the total Hilbert space, not affecting the structure of the physical subspace [14]. Then, the new effective Lagrangian, after integrating out the $\theta$ fields, becomes

$$
\mathcal{L}_{e f f}=-\frac{1}{4} F_{\mu \nu}\left(1+\frac{m^{2}}{\square}\right) F^{\mu \nu}-A_{0} J^{0},
$$

one immediately sees that $S U(2)$ Yang-Mills theory has been mapped into a $U(1)$ gauge theory.

Having established the new effective Lagrangian, we can now compute the interaction energy. To this end, we first consider the Hamiltonian framework of this new effective theory. The canonical momenta read $\Pi^{\mu}=-\left(1+\frac{m^{2}}{\square}\right) F^{0 \mu}$. This yields the usual primary constraint $\Pi^{0}=0$, and $\Pi^{i}=-\left(1+\frac{m^{2}}{\square}\right) F^{0 i}$. Therefore the canonical Hamiltonian takes the form

$$
H_{C}=\int d^{2} x\left\{-A_{0}\left(\partial_{i} \Pi^{i}-J^{0}\right)-\frac{1}{2} \Pi_{i}\left(1+\frac{m^{2}}{\square}\right)^{-1} \Pi^{i}+\frac{1}{4} F_{i j} F^{i j}\right\} .
$$

Temporal conservation of the primary constraint $\Pi_{0}$ leads to the secondary constraint $\Gamma_{1}(x) \equiv \partial_{i} \Pi^{i}-J^{0}=0$. It is straightforward to check that there are no further constraints in the theory. The extended Hamiltonian that generates translations in time then reads $H=H_{C}+\int d x\left(c_{0}(x) \Pi_{0}(x)+c_{1}(x) \Gamma_{1}(x)\right)$, where $c_{0}(x)$ and $c_{1}(x)$ are the Lagrange multipliers. Moreover, it follows from this Hamiltonian that $\dot{A}_{0}(x)=\left[A_{0}(x), H\right]=c_{0}(x)$, which is an arbitrary function. Since $\Pi_{0}=0$, neither $A^{0}$ nor $\Pi^{0}$ are of interest in describing the system and may be discarded from the theory. As a result, the Hamiltonian becomes

$$
H=\int d^{2} x\left\{-\frac{1}{2} \Pi_{i}\left(1+\frac{m^{2}}{\square}\right)^{-1} \Pi^{i}+\frac{1}{4} F_{i j} F^{i j}+c^{\prime}\left(\partial_{i} \Pi^{i}-J^{0}\right)\right\},
$$

where $c^{\prime}(x)=c_{1}(x)-A_{0}(x)$.

Since there is one first class constraint $\Gamma_{1}(x)$ (Gauss' law), we choose one gauge fixing condition that will make the full set of constraints becomes second class. We choose the gauge fixing condition to correspond to [13, 16]

$$
\Gamma_{2}(x) \equiv \int_{C_{\xi x}} d z^{\nu} A_{\nu}(z) \equiv \int_{0}^{1} d \lambda x^{i} A_{i}(\lambda x)=0,
$$

where $\lambda(0 \leq \lambda \leq 1)$ is the parameter describing the spacelike straight path between the reference points $\xi^{k}$ and $x^{k}$, on a fixed time slice. For simplicity we have assumed the reference point $\xi^{k}=0$. The choice (12) leads to the Poincaré gauge. With this we obtain the only nontrivial Dirac bracket

$$
\left\{A_{i}(x), \Pi^{j}(y)\right\}^{*}=\delta_{i}^{j} \delta^{(2)}(x-y)-\frac{\partial}{\partial x^{i}} \int_{0}^{1} d \lambda x^{j} \delta^{(2)}(\lambda x-y) .
$$

We pass now to the calculation of the interaction energy, where a fermion is localized at the origin $\mathbf{0}$ and an antifermion at $\mathbf{y}$. As already mentioned, we will calculate the expectation value of the energy operator $H$ in the physical state $|\Phi\rangle$, which we will denote by $\langle H\rangle_{\Phi}$. From our above discussion, we see that $\langle H\rangle_{\Phi} \operatorname{reads}$

$$
\langle H\rangle_{\Phi}=\left\langle\Phi\left|\int d^{2} x\left(-\frac{1}{2} \Pi_{i}\left(1+\frac{m^{2}}{\square}\right)^{-1} \Pi^{i}+\frac{1}{4} F_{i j} F^{i j}\right)\right| \Phi\right\rangle .
$$


Since the fermions are taken to be infinitely massive (static), this can be further simplified as

$$
\langle H\rangle_{\Phi}=\left\langle\Phi\left|\int d^{2} x\left(-\frac{1}{2} \Pi_{i}\left(1-\frac{m^{2}}{\nabla^{2}}\right)^{-1} \Pi^{i}\right)\right| \Phi\right\rangle .
$$

Let us also mention here that, as was first established by Dirac [19], the physical states $|\Phi\rangle$ correspond to the gauge invariant ones. It is helpful to recall at this stage that in the Abelian case $|\Phi\rangle$ may be written as [17]

$$
|\Phi\rangle \equiv|\bar{\Psi}(\mathbf{y}) \Psi(\mathbf{0})\rangle=\bar{\psi}(\mathbf{y}) \exp \left(i g \int_{\mathbf{0}}^{\mathbf{y}} d z^{i} A_{i}(z)\right) \psi(\mathbf{0})|0\rangle,
$$

where $|0\rangle$ is the physical vacuum state and the line integral appearing in the above expression is along a spacelike path starting at $\mathbf{0}$ and ending at $\mathbf{y}$, on a fixed time slice. It is to be observed that the strings between fermions have been introduced in order to have a gauge-invariant function $|\Phi\rangle$. Another way of saying the same thing is that the fermion fields are now dressed by a cloud of gauge fields.

Returning now to our problem on hand, we compute the expectation value of $H$ (given by the expression (15)) in the physical state $|\Phi\rangle$. Taking into account the above Hamiltonian structure, we observe that

$$
\Pi_{i}(x)|\bar{\Psi}(y) \Psi(0)\rangle=\bar{\Psi}(y) \Psi(0) \Pi_{i}(x)|0\rangle-e \int_{\mathbf{0}}^{\mathbf{y}} d z_{i} \delta^{(2)}(z-x)|\Phi\rangle .
$$

Inserting this back into (15), we get

$$
\langle H\rangle_{\Phi}=\langle H\rangle_{0}-\frac{e^{2}}{2 \pi} K_{0}(m L),
$$

where $\langle H\rangle_{0}=\langle 0|H| 0\rangle$ and with $|\mathbf{y}| \equiv L$. Since the potential is given by the term of the energy which depends on the separation of the two fermions, from the expression (18) we obtain

$$
V=-\frac{e^{2}}{2 \pi} K_{0}(m L)
$$

In this way the static interaction between fermions arises only because of the requirement that the $|\bar{\Psi} \Psi\rangle$ states be gauge invariant. It is interesting to note that this is exactly the result obtained for the Maxwell-Chern-Simons theory $[20,21]$.

We are now in position to examine the mapping of the theory (9) into the massive Schwinger model. Let us illustrate this by making a dimensional compactification (à la Kaluza-Klein) on Eq.(9). The compactification is needed also in order to control the infrared behavior of the theory. In such a case, the new theory takes the form:

$$
\mathcal{L}^{(1+1)}=-\frac{1}{4} F_{\mu \nu} \sum_{n}\left(1+\frac{g^{2} C^{2}}{\square(1+1)+a^{2}}\right) F^{\mu \nu}-A_{0} J^{0},
$$

where $a^{2} \equiv n^{2} / R^{2}$, and $R$ is the compactification radius. We immediately recognize the above to be the massive Schwinger model with mass $m^{2} \equiv a^{2}$. We observe, as an appealing feature of this expression, that for the zero mode $(a=0)$ case it gives the massless Schwinger model. It is now once again straightforward to compute the interaction energy. Taking a contribution of a single mode in Eq.(20), we obtain [12]:

$$
V=\frac{e^{2}}{2 \lambda}\left(1+\frac{a^{2}}{\lambda^{2}}\right)\left(1-e^{-\lambda L}\right)+\frac{e^{2}}{2}\left(1-\frac{g^{2} C^{2}}{\lambda^{2}}\right) L,
$$

where $\lambda^{2} \equiv g^{2} C^{2}+a^{2}$ and $|y| \equiv L$. Effectively, therefore, our initial theory (1) is mapped into the massive Schwinger model, which displays both the screening and the confining part of this interaction. Of course, if we consider the zero mode case, i. e. , $a=0$, the static potential above shows that confinement disappears. When studying this expression, we will concentrate on the second term of Eq. (21), which represents confinement. We start considering the subtle points related to the calculation of this confinement term. Although, as we see from Eq. (21), every single mode contribution shows confinement, one can ask the question of how to handle the sum over all modes in (21). If we are interested in the interaction potential between two point-like sources in the $(2+1)$-dimensional case (and later we will consider also the $(3+1)$-dimensional case) we must sum over all the modes with equal weight, according to 
the representation of the delta function source.

Basically, the expression for the coefficient of the linear potential between two static point sources is:

$$
T=\frac{e^{2}}{2} \sum_{n} \frac{n^{2} / R^{2}}{g^{2} C^{2}+n^{2} / R^{2}} .
$$

In the limit $R \rightarrow \infty$, the sum can be approximated by an integral. Defining the continuous variable $x=\frac{n}{R}$, Eq. (22) can be rewritten as

$$
T=\frac{e^{2}}{2} R \int_{0}^{\Lambda} d x \frac{x^{2}}{g^{2} C^{2}+x^{2}},
$$

where $\Lambda$ is an ultraviolet cutoff. After some manipulations, we get that $T$ is given by

$$
T=\frac{e^{2} R}{2}\left(\Lambda-\frac{\pi}{4} g C\right),
$$

From expression (24), in the limit $R \rightarrow \infty$, the only way to obtain a finite value for $T$ is to let $C \rightarrow \infty$ according to

$$
C \rightarrow \frac{4 \Lambda}{\pi g}+\frac{\kappa}{R}
$$

where $\kappa$ is a constant which determines the value of the constant $T$. In other words, the external background field (3) has to be selected of the order of the ultraviolet cutoff. This is in complete accordance with the result of [5], where when considering the cancellation of infrared divergences one has to consider constant external gauge fields of infinite value as the cutoff is removed.

At this stage, it is interesting to recall the results reported in Refs. [22, 23] for a $(2+1)$-dimensional $S U(N)$ Yang-Mills theory

$$
V(L)=\frac{g^{2} C_{F}}{2 \pi} \log \left(g^{2} L\right)+\frac{7}{64 \pi} g^{4} C_{F} C_{A} L,
$$

where $C_{F}$ and $C_{A}$ are the Casimir group factor. Hence we see that our phenomenological result (21) agrees qualitatively with (26) in the limit of large $L$. Corroborating that the reformulation of $S U(2)$ Yang-Mills theory in terms of the new set of variables is suitable for its low energy content. In this way the massive Schwinger model simulates the features of the $S U(2)$ Yang-Mills theory. Notice that in our teatment, as $C \rightarrow \infty$ the first term in (21) (even after summation) does not lead to a non trivial contribution.

\section{FINAL REMARKS}

In summary, we have considered the confinement versus screening issue for a reformulated three-dimensional YangMills theory. This reformulation is achieved due to the presence of condensates of gauge fields, which are implemented by a new set of variables. In terms of these variables the long distance physics is described by an effective action which is a $U(1)$ gauge theory leading to a linear potential between static charges.

In the $(3+1)$-dimensional case, we can perform the compactification of two spatial dimensions, assigning to them radii $R_{1}$ and $R_{2}$, we obtain for the coefficient of the linear potential the expression

$$
T=\frac{e^{2}}{2} \sum_{n_{1}, n_{2}} \frac{\left(\frac{n_{1}^{2}}{R_{1}^{2}}+\frac{n_{2}^{2}}{R_{2}^{2}}\right)}{g^{2} C^{2}+\frac{n_{1}^{2}}{R_{1}^{2}}+\frac{n_{2}^{2}}{R_{2}^{2}}} .
$$

Following the same steps of the $(2+1)$-dimensional case, in the limit $R_{1}, R_{2} \rightarrow \infty$ we obtain

$$
T=\pi e^{2} R_{1} R_{2} \int_{0}^{\Lambda} d \rho \frac{\rho^{3}}{C^{2}+\rho^{2}}
$$

that is,

$$
T=\frac{\pi e^{2}}{2} R_{1} R_{2}\left[\Lambda^{2}-g^{2} C^{2} \ln \left(\frac{g^{2} C^{2}+\Lambda^{2}}{g^{2} C^{2}}\right)\right],
$$


again if $R_{1}, R_{2} \rightarrow \infty$, we obtain the transcendental equation for $\Lambda / g^{2} C^{2}$ :

$$
\frac{\Lambda^{2}}{g^{2} C^{2}}-\ln \left(1+\frac{\Lambda^{2}}{g^{2} C^{2}}\right)=0
$$

From (29) here we can deduce that as $\frac{\Lambda^{2}}{g^{2} C^{2}} \sim \sqrt{\frac{2 \pi T}{e^{2} R_{1} R_{2}}} \rightarrow 0$, which means that the external field $C$ has to grow stronger than $g \Lambda$ when $\Lambda \rightarrow \infty$, in order to obtain a finite coefficient of the linear potential.

Although this method appears simple and intuitive, it displays the basic features of how a confining potential can appear even in the $(3+1)$-dimensional gauge theory. Finally, notice that once the space has undergone a compactification, the background field (3) may not be transformed away by a gauge transformation, since such a gauge transformation in general would violate the periodicity conditions. Another way of understanding the physical reality of the background (3) is by calculating the gauge invariant Wilson loops of (3) along the compactified dimensions, which are non vanishing.

\section{ACKNOWLEDGMENTS}

One of us (E. G.) wants to thank the Physics Department of the Universidad Técnica F. Santa María for hospitality. P. G. was partially supported by FONDECYT (Chile) grant 1050546.

[1] S. Capstick et al., Key issues in hadronic physics, [hep-ph/0012238].

[2] G. K. Savvidy, Phys. Lett. B71, 133 (1977).

[3] H. B. Nielsen and P. Olesen, Nucl. Phys. B152, 75 (1979); N. K. Nielsen and P. Olesen, Nucl. Phys. B144, 376 (1978).

[4] P. Gaete, E. Spallucci J. Phys. A39, 6021 (2006)

[5] E. I. Guendelman, Int. J. Mod. Phys. A5, 4341 (1990).

[6] E. I. Guendelman and D. A. Owen, Mod. Phys. Lett. A6, 41 (1991). For related works on infrared divergences in (2+1)dimensions theories see also E. I. Guendelman and Z. M. Radulovic, Phys. Rev. D27, 357 (1983); Phys. Rev. D30, 1338 (1984).

[7] M. Luscher, Phys. Lett. B78, 465 (1978)

[8] G. Gabadadze Phys. Rev. D58, 094015 (1998);

[9] K. Kondo, Phys. Rev. D57, 7467 (1998)

[10] A. Aurilia, E. Spallucci Phys.Rev. D69 , 105004 (2004) A. Aurilia, E. Spallucci Phys.Rev. D69 , 105005 (2004)

[11] J. Schwinger, Phys. Rev. 128, 2425 (1962).

[12] P. Gaete and I. Schmidt, Phys. Rev. D61, 125002 (2000).

[13] P. Gaete, Z. Phys. C76, 355 (1997).

[14] C. Wotzasek, Int. J. Mod. Phys. A5, 1123 (1990).

[15] N. Banerjee and R. Banerjee, Mod. Phys. Lett. A11, 1919 (1996).

[16] P. Gaete and E. I. Guendelman, Phys. Lett. B593, 151 (2004).

[17] P. Gaete, Phys. Lett. B515, 382 (2001).

[18] P. Gaete, Phys. Lett B582, 270 (2004).

[19] P. A. M. Dirac, The Principles of Quantum Mechanics (Oxford University Press, Oxford, 1958); Can. J. Phys. 33, 650 (1955).

[20] E. Abdalla and R. Banerjee, Phys. Rev. Lett. 80, 238 (1998).

[21] P. Gaete, Phys. Rev. D59, 127702 (1999).

[22] Y. Schröeder, Phys. Lett. B447, 321 (1999).

[23] E. Bagan, M. Lavelle and D. McMullan, Phys. Lett. B477, 355 (2000). 